\title{
Impact of Co-Morbidities in Non Alcoholic Fatty Liver Disease among Type 2 Diabetes Mellitus
}

\author{
Anusri $\mathbf{T}^{1}$, Lisa Koshy ${ }^{2}$, Vinod Thomas ${ }^{3}$
}

\begin{abstract}
Non-alcoholic liver disease(NAFLD) is a common liver disorder which is closely associated with insulin resistance and type 2 diabetes mellitus and it is characterised by fat accumulation in the liver.The prevalence in diabetic population is found to be 20-40\%. Studies have shown that NAFLD is strongly associated with severalmetabolic disordersand diseases, such as obesity, type 2 diabetes mellitus, and dyslipidemia. This review article aim to check the association of co-morbidities in non-alcoholic fatty liver among type 2diabetes mellitus. NAFLD deserves particular attention given that NAFLD could be a risk factor for the development of metabolic syndrome, $C V D$, and $C K D$.
\end{abstract}

Keywords: Non-alcoholic fatty liver disease, Type 2diabetes mellitus, Dyslipidemia, Obesity, Hypertension

\section{Introduction}

Non-alcoholic fatty liver disease (NAFLD) is an upcoming cause of chronic liver disease worldwide and is becoming a major health problem in current scenario. NAFLDis considered as a spectrum of liver conditions from simple non-alcoholic steato hepatitis (NASH) to advanced hepatic fibrosis. In other wordsit is defined as a liver disorder which is accompanied with insulin resistance and type 2 diabetes, and characterized by fatty accumulation in the liver.[1,2]

Liver disease is one of the growing cause of death in type 2 diabetes. In a population-based diabetes study, cirrhosis was found to be the fourth leading cause of death in diabetes related deaths. NAFLD is a clinicalpathologic disorder that is related to obesity, dyslipidemia, cardiovascular disease and type 2 diabetes[3-5].

The prevalence of nonalcoholic fatty liver, is greater in manyobesity-related disorders, and it has been shown that there is an increased risk for NALFD of obese persons with a body-mass index (BMI) of a least $30 \mathrm{~kg} / \mathrm{m}^{2}$. Type 2 diabetes increases not only the risk of NAFLD but also the severity of the disease regardless of BMI .The prevalence of NAFLD in T2DM is approximately $50 \%$ in the US.In patients with T2DM and severe obesity 100 percent had at least mild steatosis, 50 percent had steatohepatitis and 19 percent had cirrhosis. Insulin resistance and hyperinsulinemia are also related with NAFLD in subjects without T2DM. More over, patients with a history of T2DM are atincreased risk to develop NASH. Hypertriglyceridemia, without hypercholesterolemia,is also an important risk factor for NAFLD[6].

Although the exact pathogenesis of NAFLD is unknown, the prevailing hypothesis by is that several "hits" are involved in causing progressive liver injury. Fromthe initial hit, macro vesicular steatosis results. Insulin resistance plays a important role in the net retention of lipids, particularly triglycerides, within the hepatocytes. Even though the mechanisms have not been completely elucidated, it is thought to result from decreased disposalof fatty acids due to impaired mitochondrial $\beta$-oxidation. The second hit is related to oxidative stress, it causes peroxidation of lipids in the hepatocyte membrane, cytokine production, and Fas ligand induction and is responsible for the progression from steatosisto NASH to cirrhosis. Bacterial toxins, overproduction of cytokines (especially tumor necrosis factor- $\alpha$ ), and alteration of hepatocyte ATP stores and cytochrome P450 Cyp2E1/Cyp4A enzyme activity are also precipitating factor for disease progression and fibro genesis. The elevated serum leptinmay promote hepatic steatosis and steatohepatitis, correlate directly with severity of hepatic steatosis without inflammation or fibrosis .[7]

\section{Hypertension and NAFLD}

Essential hypertension is largely associated with metabolic syndrome; hyperinsulineamia being seen in up to $50 \%$ of non-obese patients with hypertension. Abdominal obesity which is central risk factor for the development of hypertension is also associated with insulin resistance and metabolic syndrome. A relationship between abnormal liver function test and hypertension was identified in a previous study and found that up to $15 \%$ of all male hypertensive patients had abnormal liver function test.[8] A recent prospective study has confirmed a positive association between age, serum alpha glutamyl transferase and the following risk of hypertension. [9] But hypertension has been correlated with the development of severe NAFLD in obese patients, hypertension and fatty liver have also being linked in the non-obese patients.[10] Another recent study shows that hypertensive patients have a greater extent of prevalence in NAFLD and it is independently associated with hypertension and blood pressure category.[11]

\section{Dyslipidaemia and hypertension}

The exact cause of NAFLD with dyslipidaemia is poorly sunderstood. Nevertheless, it can be justified that hepatic steatosis is a result of net hepatocellualr retension of lipids, especially in the form of triglycerides. From a liver centre point of view this imbalance results from abnormalities in one or more of the following four process :

- Hepatic uptake of fatty acid, Lipoprotein and glucose 


\section{International Journal of Science and Research (IJSR) \\ ISSN (Online): 2319-7064}

Index Copernicus Value (2015): 78.96 | Impact Factor (2015): 6.391

- De novo triglyceride synthesis

- TG degradation and fatty acid beta oxidation

- Lipoprotein secretion in the form of VLDL.[12]

Dyslipidemia that is characterized by high triglyceride (TG) and low high-density lipoprotein cholesterol (HDL-C) levels predisposes patients to arthrosclerosis. [13] Almost 20-80\% of NAFLD patients also have dyslipidemia.[14] A common change in the metabolic profile among patients with T2DM, MS, and obesity is an alteration of serum lipid levels (dyslipidemia),suggestingacloserelationshipbetweenT2DM,

MS, and obesity and NAFLD. It has been shown that NASH significantly boosts the level of oxidized low-density lipoprotein cholesterol (LDL-C). High LDL-C is a wellknown risk factor for arthrosclerosis.[15] The most common form of dyslipidemia in NAFLD patients is atherogenic dyslipidemia, which is characterized by hypertriglyceridemia, low HDL-C levels, and high LDL-C levels.[16] Long-term dyslipidemia may increase the expression and activity of sterol regulatory element binding protein-1c, a transcription factor, which adversely affects the profiles of lipid and lipoprotein synthesis in the liver, including increased TG, LDL, and very low-density lipoprotein (VLDL) levels and decreased HDL-C levels.[17,18] There is a strong link between insulin resistance and metabolic dyslipidemia in T2DM. Insulin resistance facilitates the increase of free fatty acid (FFA) flux. The increased FFA level boosts TG and VLDL release as well as triggers oxidative stress and lipid peroxidation, all of which are related with the development of NAFLD.[19,20]

\section{Obesity and NAFLD}

The reason for fat deposition in the liver among NAFLD remains unknown. However, adipose tissue releases adipocytes, which may bean important factor that increases liver fat content . Manifestation of the liver to free fatty acids may be another important factor and it could be due to dietary fat intake and release of free fatty acids, especially from abdominal adipose tissue [21,22]. Abdominal adipose tissue includes distinct anatomic depots, a subcutaneous fat depot and an intraabdominal fat depot, which can be divided into intraperitoneal and retroperitoneal depots. The intraperitoneal fat depot, also known as visceral fat, can be divided into mesenteric and omental depots [23]. Subcutaneous fat differs from visceral fat in that venous drainage from subcutaneous fat is directed into the systemic circulation, whereas venous drainage from visceral fat is directed into the portal vein. The metabolic products thus reach the liver directly and exercise a first-pass effect on liver metabolism. It has been hypothesized that visceral fat releases free fatty acids and adipokines and thereby exposes the liver to fat deposition [24,25]. Release from visceral fat of free fatty acids transported through the portal vein to the liver is promoted by an investigation conducted in a study. However, even in viscerally obese persons, more than 50-60 percent of the delivery of free fatty acids to the liver comes from the systemic circulation [26].

\section{Chronic kidney disease and NAFLD}

Currently, many studies have reported that metabolic syndrome and insulin resistance are associated with greater incidence in micro albuminuria and chronic kidney disease (CKD) [27-31]. CKD and NAFLD were connected to the cardiometabolic risk factors including metabolic syndrome and its individual elements [32-34]. Interrelations between NAFLD, insulin resistance, and metabolic syndrome increase the possibility that NAFLD can figure out the development and progression of CKD [35-38]. Because of study population heterogeneity, and differences in NAFLD diagnostic features, NAFLD severity, and CKD definition used, the reported prevalence and incidence of CKD are largely conflicting. However, most of the recent large population based andhospital based studies represent that the prevalence and incidence of CKD was greatly increase in subjects with NAFLD [39-44]. According to a current metaanalysis,NAFLD was connected with an raised prevalence and incidence of CKD and nonalcoholic steatohepatitis(NASH) was related with a higher prevalenceand incidence of CKD than simple steatosis independent of potential confounding factors including age, smoking, obesity, hypertension, and metabolic syndrome elements [45].

\section{Conclusion}

The prevalence of non-alcoholic fatty liverdisease is continuously increasing around the world and NAFLD is becoming a pandemic disease in concert with the on-going epidemics of obesity, diabetes, and metabolic syndrome.NAFLD is connected with a moderately rised risk for future CVD events among type 2 diabetic subjects, independent of classical risk factors, liver enzymes, and the presence of metabolic syndrome. Growing evidence suggests that NAFLD is associated with metabolic derangement and other systemic morbidities. moreover, currently NAFLD has been identified as an independent risk factor for metabolic syndrome, type 2 diabetes mellitus, CVD, and CKD and the severity of NAFLD is associated with disease manifestations. NAFLD deserves particular attention given that NAFLD could be a risk factor for the development of metabolic syndrome, CVD, and CKD.

\section{Acknowledgment}

This review article is a part of our academic project. We thank Dr. Anoop Kumar MD, Dept. of General Medicine for his thoughtful comments on the manuscript. Mrs. Jereena E in the preparation of the manuscript.

\section{References}

[1] Comorbidities and Metabolic Derangement of NAFLD $\mathrm{Ki}$ Bae Bang and Yong Kyun Cho*,Department of Internal Medicine, Kangbuk Samsung Hospital, Sungkyunkwan University School of Medicine, Seoul, Korea, Journal of Lifestyle Medicine 2015;5:7-13.

[2] Vijay Viswanathan, Mahesh Kadiri, Srikanth Medimpudi, Satyavani Kumpatla. Association of nonalcoholic fatty liver disease with diabetic microvascular 


\section{International Journal of Science and Research (IJSR) \\ ISSN (Online): 2319-7064}

Index Copernicus Value (2015): 78.96 | Impact Factor (2015): 6.391

and macrovascular complications in South Indian diabetic subjects. International Journal of Diabetes in Developing Countries 2010; 30(4):208-212.

[3] Keith G. Tolman, ,Vivian Fonseca, Anthony Dalpiaz. Spectrum of Liver Disease in Type 2Diabetes and Management of Patients With Diabetes and Liver DiseaseCorrelation Of Non-Alcoholic Fatty Liver Diseaseand Features Of Metabolic Syndrome In Morbidly Obese Patients, Diabetes care;30(3):734-743.

[4] Fernando de Barros, Sergio Setúbal, José Manoel Martinho, Loraine Ferraz, Andressa Gaudêncio. In The Preoperative Assessment For Bariatric Surgery, ABCD Arq Bras Cir Dig 2016;29(4):260-263.

[5] Giovanni Targher, Lorenzo Bertolini, Felice Poli, Stefano Rodella, Luca Scala, Roberto Tessari, Luciano Zenari, and Giancarlo Falezza. Non-alcoholic Fatty Liver Disease and Risk of Future Cardiovascular Events Among Type 2 Diabetic Patients, Diabetes J2005;54:3541-3546.

[6] Ronald J.H. Borra,Non-Alcoholic Fatty Liver Disease In Obesity And Type 2 Diabetes Studies using 1H MRS and PET. Medical Imaging Centre of Southwest Finland and Turku PET Centre; Department of Diagnostic Radiology, University of Turku, Finland AnnalesUniversitatisTurkuensis, PainosalamaOy, Turku, Finland 2009:17-18.

[7] David A. Sass,Parke Chang, and Kapil B. Chopra, Nonalcoholic Fatty Liver Disease: A Clinical Review, Digestive Diseases asnd Sciences2005;50:171-180

[8] M.J. Brookes, B.T. Cooper.Hypertension and fattyliver: Guilty by association. Journal of human hypertension;2011;21:264-270.

[9] Lee DH, Ha MH, Kim KY, Jin DG, Jacobs Jr Dr., Gamma glutamyl transferase: An Effect modifier in the association between age and hypertension in a4 year follow up study. J Hum Hypertens 2004;18:803-807.

[10]Dixon JB, Bhathal PS, O'Brien PE. Non-alcoholic fattyliver disease: Predictors of Non alcoholic steatohepatitis and liver fibrosis in severly obese. Gastroenterology 2001;121:91-100.

[11] Yaohui wang, Yuhua zeng, Changwei Lin, Zhiheng Chen. Hypertension and non-alcoholic fatty liver disease proven by transient elastography. Hepatology reaserch 2016;:46: 1304-1310.

[12] Angulo P., Non alcoholic fatty liver disease. New England Journal med 2002;346:1221-1231.

[13] Targher G, Day CP, Bonora E. Risk of cardiovascular disease in patients with nonalcoholic fatty liver disease. $\mathrm{N}$ Engl J Med 2010;363:1341-1350. doi: 10.1056/NEJMra0912063.

[14] Souza MR, Diniz MF, Medeiros JE, Araujo MS. Metabolic syndrome and risk factors for non-alcoholic fatty liver disease. Arq Gastroenterol 2012;49:89-96.

[15] Norris AL, Steinberger J, Steffen LM, Metzig AM, Schwarzenberg SJ, Kelly AS. Circulating oxidized LDL and inflammation in extreme pediatric obesity. Obesity 2011;19:1415-1419. doi: 10.1038/oby.2011.21.

[16] Sahebkar A1, Chew GT, Watts GF. New peroxisome proliferator-activated receptor agonists: potential treatments for atherogenic dyslipidemia and nonalcoholic fatty liver disease. Expert Opin Pharmacother 2014, 15:493- 503. doi: $10.1517 / 14656566.2014 .876992$.
[17] Ahmed MH, Abu EO, Byrne CD. Non-Alcoholic Fatty Liver Disease (NAFLD): new challenge for general practitioners and important burden for health authorities? Prim Care Diabetes 2010;4:129-137. doi: 10.1016/j.pcd.2010. 02.004.

[18] Gawrieh S, Baye TM, Carless M, Wallace J, Komorowski R, Kleiner DE, et al. Hepatic gene networks in morbidly obese patients with nonalcoholic fatty liver disease. Obes Surg 2010;20:1698-1709. doi: 10.1007/s11695-0100171-6.

[19] Alkhouri N, Eng K, Lopez R, Nobili V. Non-highdensity lipoprotein cholesterol (non-HDL-C) levels in children with nonalcoholic fatty liver disease (NAFLD). Springerplus 2014;3:407. doi: 10.1186/2193-1801-3407.

[20] Cao W, Zhao C, Shen C, Wang Y. Cytokeratin 18, alanine aminotransferase, platelets and triglycerides predict the presence of nonalcoholic steatohepatitis. PLOS One 2013;8:e82092. doi: 10.1371/journal.pone.0082092.

[21] Yki-Jarvinen H. Fat in the liver and insulin resistance. Ann Med 2005;37:347-56.

[22] Westerbacka J, Lammi K, Hakkinen AM, et al. Dietary fat content modifies liver fat in overweight nondiabetic subjects. J Clin Endocrinol Metab 2005;90:2804-9.

[23] Frayn KN. Visceral fat and insulin resistancecausative or correlative? Br J Nutr 2000;83(suppl 1):S71-7.

[24] Engfeldt P, Arner P. Lipolysis in human adipocytes, effects of cell size, age and of regional differences. Horm Metab Res Suppl 1988;19:26-9.

[25] Bjorntorp P. "Portal' adipose tissue as a generator of risk factors for cardiovascular disease and diabetes. Arteriosclerosis 1990;10:493-6.

[26] Nielsen S, Guo Z, Johnson CM, et al. Splanchnic lipolysis in human obesity. $\mathrm{J}$ Clin Invest 2004;113:1582-8.

[27] Satchell SC, Tooke JE. What is the mechanism of microalbuminuria in diabetes: a role for the glomerular endothelium? Diabetologia 2008;51:714-25.

[28] Chen J, Muntner P, Hamm LL, Jones DW, Batuman V, Fonseca V, Whelton PK, He J. The metabolic syndrome and chronic kidney disease in U.S. adults. Ann Intern Med 2004;140:167-74.

[29] Kurella M, Lo JC, Chertow GM. Metabolic syndrome and the risk for chronic kidney disease among nondiabetic adults. J Am Soc Nephrol 2005;16:2134-40.

[30] Chen J, Muntner P, Hamm LL, Fonseca V, Batuman V, Whelton PK, He J. Insulin resistance and risk of chronic kidney disease in nondiabetic US adults. J Am Soc Nephrol 2003;14:469-77.

[31] Lin CC, Liu CS, Li TC, Chen CC, Li CI, Lin WY. Microalbuminuria and the metabolic syndrome and its components in the Chinese population. Eur J Clin Invest 2007;37:783-90.

[32] Hwang ST, Cho YK, Yun JW, Park JH, Kim HJ, Park DI, Sohn CI, Jeon WK, Kim BI, Rhee EJ, Oh KW, Lee WY, Jin W. Impact of non-alcoholic fatty liver disease on microalbuminuria in patients with prediabetes and diabetes. Intern Med J 2010;40:437-42.

[33] Stefan N, Kantartzis K, Haring HU. Causes and metabolic consequences of Fatty liver. Endocr Rev 2008;29: 939-60. 


\section{International Journal of Science and Research (IJSR) \\ ISSN (Online): 2319-7064 \\ Index Copernicus Value (2015): 78.96 | Impact Factor (2015): 6.391}

[34] Targher G, Chonchol M, Zoppini G, Abaterusso C, Bonora E. Risk of chronic kidney disease in patients with non-alcoholic fatty liver disease: is there a link? J Hepatol 2011;54:1020-9.

[35] Sesti G, Fiorentino TV, Arturi F, Perticone M, Sciacqua A, Perticone F. Association between noninvasive fibrosis markers and chronic kidney disease among adults with nonalcoholic fatty liver disease. PLoS One 2014;9: e88569.

[36] Lee DH, Jacobs DR, Jr., Gross M, Steffes M. Serum gamma-glutamyltransferase was differently associated with microalbuminuria by status of hypertension or diabetes: the Coronary Artery Risk Development in Young Adults (CARDIA) Study. Clin Chem 2005;51: 1185-91.

[37] Ryu S, Chang Y, Kim DI, Kim WS, Suh BS. gammaGlutamyltransferase as a predictor of chronic kidney disease in nonhypertensive and nondiabetic Korean men. Clin Chem 2007;53:71-7.

[38] Chang Y, Ryu S, Sung E, Woo HY, Oh E, Cha K, Jung E, Kim WS. Nonalcoholic fatty liver disease predicts chronic kidney disease in nonhypertensive and nondiabetic Korean men. Metabolism 2008;57:569-76.

[39] Targher G, Chonchol M, Bertolini L, Rodella S, Zenari L, Lippi G, Franchini M, Zoppini G, Muggeo M. Increased risk of CKD among type 2 diabetics with nonalcoholic fatty liver disease. J Am Soc Nephrol 2008; 19:1564-70.

[40] Yilmaz Y, Alahdab YO, Yonal O, Kurt R, Kedrah AE, Celikel CA, Ozdogan O, Duman D, Imeryuz N, Avsar E, Kalayci C. Microalbuminuria in nondiabetic patients with nonalcoholic fatty liver disease: association with liver fibrosis. Metabolism 2010;59:1327-30.

[41] Targher G, Bertolini L, Rodella S, Lippi G, Zoppini G, Chonchol M. Relationship between kidney function and liver histology in subjects with non-alcoholic steatohepatitis. Clin J Am Soc Nephrol 2010;5:2166-71.

[42] Ahn AL, Choi JK, Kim MN, Kim SA, Oh EJ, Kweon HJ, Cho DY. Non-alcoholic Fatty Liver Disease and Chronic Kidney Disease in Koreans Aged 50 Years or Older. Korean J Fam Med 2013;34:199-205.

[43] Casoinic F, Sampelean D, Badau C, Pruna L. Nonalcoholic fatty liver disease--a risk factor for microalbuminuria in type 2 diabetic patients. Rom J Intern Med 2009;47:55-9.

[44] Targher G, Pichiri I, Zoppini G, Trombetta M, Bonora E. Increased prevalence of chronic kidney disease in patients with Type 1 diabetes and non-alcoholic fatty liver. Diabet Med 2012;29:220-6.

[45] Musso G, Gambino R, Tabibian JH, Ekstedt M, Kechagias S, Hamaguchi M, Hultcrantz R, Hagstrom H, Yoon SK, Charatcharoenwitthaya P, George J, Barrera F, Hafliethadottir S, Bjornsson ES, Armstrong MJ, et al. Association of non-alcoholic fatty liver disease with chronic kidney disease: a systematic review and metaanalysis. PLoS Med 2014;11:e1001680. 